\title{
¿La altura protege frente a la COVID-19?
}

\section{Does high altitude protect against COVID-19?}

\author{
Cristhian Adolfo Vizcarra-Vizcarra '
}

Sr. editor:

La COVID-19 produce un síndrome respiratorio agudo grave caracterizado por neumonía viral, insuficiencia respiratoria y falla multiorgánica con mortalidad elevada. Tiene mayor prevalencia en el sexo masculino debido a factores como la influencia hormonal de la testosterona que permite la mayor expresión de la ECA-2 y que la proteína serina-2 (TMPRSS2) se expresa predominantemente en el tejido prostático y su transcripción está regulada por ligandos androgénicos. ${ }^{1}$ Resaltan las comorbilidades en la mayoría de pacientes, entre ellas hipertensión arterial, diabetes mellitus, obesidad, cáncer, inmunosupresión y la enfermedad renal crónica. ${ }^{2,3}$

A inicios de la pandemia, se mencionó un papel protector de la altura frente a la COVID-19 al encontrarse menor incidencia de casos en ciudades por encima de los 2500 metros sobre el nivel del mar (msnm), lo cual podría estar mediado por la mayor radiación ultravioleta y por una disminución de la expresión de ECA-2 secundario a la hipoxia crónica. ${ }^{4}$ Por esto, realizamos una búsqueda de la literatura disponible en PubMed hasta el 18 de marzo del 2021, usando los términos «COVID-19», «SARS-CoV-2» $\mathrm{y}$ «high altitude», encontrando escasos artículos referidos a COVID-19 y altura.

De hecho, no se ha podido demostrar alguna potencial relación causa-efecto o menor incidencia de COVID-19 en las poblaciones de altura y existe escasa literatura al respecto. Un estudio descriptivo que evaluó los datos de siete países con ciudades de altura de más de 100000 habitantes encontró menores tasas de mortalidad en zonas geográficas con alturas superiores a $2500 \mathrm{msnm} .{ }^{5}$ Aunque

Médico nefrólogo. Hospital III Goyeneche,Arequipa, Perú.
Vizcarra-Vizcarra CA. ¿La altura protege frente a la COVID-19?. 202।;34(2): 88-89.

https://doi.org/10.36393/spmi.v34i2.605

estos resultados parecían prometedores, por su plausibilidad biológica, debe tenerse en cuenta otros factores sociales, demográficos y e pidemiológicos q ue $\mathrm{p}$ odrían generar subregistros. ${ }^{6}$ También, se planteó una similitud entre la lesión pulmonar por COVID-19 y el edema pulmonar agudo de altitud, por lo que se sugirió el uso de acetazolamida, nifedipino e inhibidores de la fosfodiesterasa como parte del arsenal terapéutico contra el coronavirus. ${ }^{7}$ Sin embargo, cuatro publicaciones desvirtuaron esta hipótesis, ya que el mecanismo fisiopatológico e s t otalmente diferente. ${ }^{8-11}$ Zeng, en una población a 2979 msnm encontró una tasa significativamente $\mathrm{m}$ enor $\mathrm{d}$ e fi ebre y ma yor nú mero de pacientes menores de 14 años que reportes previos; además, sólo el $25 \%$ cursó con elevación de proteína $\mathrm{C}$ reactiva y cinco pacientes tuvieron una tomografía negativa al inicio y que durante el seguimiento se tornó positiva. ${ }^{12}$

En el Perú, se confirmó el primer c aso de la enfermedad el día 6 de marzo del 2020 y en el Departamento de Puno, ubicado a $3827 \mathrm{msnm}$, el día 7 de abril del mismo año; y, desde entonces, hubo miles de casos y fallecidos en nuestro país y en varias regiones de gran altitud. Algunos reportes iniciales en el país mostraron una menor incidencia y mortalidad, atribuyéndolo a la menor susceptibilidad de desarrollar efectos hipóxicos adversos graves producto de la aclimatación y adaptación fisiológica a la altura ${ }^{13}$, a una baja prevalencia de diabetes, hipertensión y obesidad en altura $^{14}$, y a factores como la menor cantidad de receptores ECA-2 y el aumento de la eritropoyetina. ${ }^{15,16}$ Estos hallazgos sólo generaron un "exceso de confianza" en la población de altura peruana, que permitió un incremento de casos al no respetar las medidas preventivas, lo que fue demostrado por Castagnetto et al, quienes concluyeron que la altura no protegía contra la infección ni la mortalidad por COVID-19. ${ }^{17}$ Además, otro punto en contra fue que, en regiones como Puno o Pasco, no se disponía de pruebas moleculares y los casos se diagnosticaban con prueba serológica, cuya baja sensibilidad y especificidad no generaron resultados confiables. ${ }^{18}$ 
En conclusión, la infección por SARS-CoV-2 ha producido una situación catastrófica a nivel mundial por el número elevado de casos y muertes que trajo consigo. A pesar de que, inicialmente, se planteó a la altura como un factor protector, los resultados son contradictorios y podemos concluir que el vivir en ciudades de gran altura, o viajar a ellas, no previene la infección por COVID-19 ni mejora la mortalidad, por lo que se debe instar a la población residente de ciudades por encima de los $2500 \mathrm{msnm}$ a no descuidar las medidas de prevención conocidas.

\section{REFERENCIAS BIBLIOGRÁFICAS}

I. Gebhard C, Regitz-Zagrosek V, Neuhauser H, Morgan R, Klein S. Impact of sex and gender on COVID-19 outcomes in Europe. Biology of Sex Differences. 2020;II(29):I-I3. URL disponible en: https://doi.org/I0.I I86/s I 3293-020-00304-9

2. Guan W, Ni Z, Hu Yu, LiangW, Ou C, He J, et al. Clinical characteristics of coronavirus disease 2019 in China.NEnglJMed.2020;382(I8): I 708I720.URL disponible en:http://dx.doi.org/I0.1056/NEJMoa2002032.

3. Richardson S, Hirsch J, Narasimhan M, Crawford J, McGinn T, Davidson K, et al. Presenting characteristics, comorbidities, and outcomes among 5700 patients hospitalized with COVID-19 in the New York City Area. JAMA. 2020:EI-E8. URL disponible en: http:// dx.doi.org/I0.100I/jama.2020.6775

4. Arias-Reyes C, Zubieta-De Urioste N, Poma-Machicao L, et al Does the pathogenesis of SARS-CoV-2 virus decrease at highaltitude? Respir Physiol Neurobiol. 2020;277:103443. URL disponible en: https://doi.org/I0.1016/j.resp.2020.103443

5. Cardona-Rivero A, Montoya M. COVID 19 en población residente de zonas geográficas a alturas superiores a 2500 m.s.n.m. SITUA. 2020: 23(I):16. [Internet]. URL disponible en: http://200.48.82.28/ index.php/SITUA/article/view/204

6. Huamaní C, Velásquez L, Montes S, Miranda-Solis F. Propagation by COVID-19 at high altitude: Cusco case [published online ahead of print, 2020 May 8]. Respir Physiol Neurobiol. 2020;279:103448. doi:10.1016/j.resp.2020.103448

7. Solaimanzadeh I. Acetazolamide, nifedipine and phosphodiesterase inhibitors: rationale for their utilization as adjunctive countermeasures in the treatment of coronavirus disease 2019 (COVID-19). Cureus. 2020; I 2(3):e7343. Published 2020 Mar 20. doi: 10.7759/cureus.7343

8. Luks AM, Freer L, Grissom CK, et al. COVID-I9 lung injury is not high-altitude pulmonary edema [published online ahead of print, 2020 Apr 13]. High Alt Med Biol. 2020; 10.1089/ham.2020.0055. doi: I0.1089/ham.2020.0055

9. Luks AM, Swenson ER. COVID-19 Lung injury and high-altitude pulmonary edema: a false equation with dangerous implications [published online ahead of print, 2020 Apr 24]. Ann Am Thorac Soc. 2020;10.15 I3/AnnalsATS.202004-327FR. doi: I0.1513/AnnalsATS. 202004-327FR
I0. Brugger H, Basnyat B, Ellerton J, Hefti U, Strapazzon G, Zafren K. COVID- 19 lung injury is different from high altitude pulmonary edema [published online ahead of print, $2020 \mathrm{Apr}$ 30]. High Alt Med Biol. 2020; I0.1089/ham.2020.006I. doi:I0.1089/ham.2020.006 I

II. Strapazzon G, Hilty MP, Bouzat P, et al.To compare the incomparable: COVID-I9 pneumonia and high-altitude disease. Eur Respir J. 2020; inpress.URLdisponibleen:https://doi.org/I0.I I83/I3993003.01362-2 020.

12. Zeng J, Peng S, Lei Y, Huang J, Guo Y, Zhang $X$, et al. Clinical and imaging features of covid- 19 patients: analysis of data from highaltitude areas. J Infect. 2020: 80(6), e34-e36. URL disponible en: https://doi.org//0.1016/j.jinf.2020.03.026

13. Canales-Gutiérrez A, Canales-Manchuria GP, Canales-Manchuria F. Adaptation to hypobaric hypoxia of residents at high altitude, to counteract COVID-19 disease. Enferm Clin. 2021 MarApr;3 I (2): |30-I31. doi: 10.1016/j.enfcli.2020.10.012

14. Seclén SN, Nunez-Robles E, Yovera-Aldana M, Arias-Chumpitaz A Incidence of COVID-19 infection and prevalence of diabetes, obesity and hypertension according to altitude in Peruvian population. Diabetes Res Clin Pract. 2020 Nov;169:108463. doi: 10.1016/j.diabres.2020.108463

15. Accinelli RA, Leon-Abarca JA. At high altitude COVID-19 is less frequent: The experience of Peru. Arch Bronconeumol. 2020 Nov;56(I I):760-76I. doi: I0.1016/j.arbres.2020.06.015

16. Millet GP, Debevec T, Brocherie F, Burtscher M, Burtscher J.Altitude and COVID-19: Friend or foe? A narrative review. Physiol Resp. 2021 Jan;8(24):e |46I5. doi: I0.|48|4/phy2.146I5

17. Castagnetto JM, Segovia-Juarez J, Gonzales GF. Letter to the Editor: COVID-19 Infections do not change with increasing altitudes from I,000 to 4,700 m. High Alt Med Biol. 2020 Dec;2I (4):428-430. doi: 10.1089/ham.2020.0173

18. Perez-Castilla J. High Altitude and COVID-19: A relationship difficult to assess. Arch Bronconeumol. 2021. https://doi.org/10.1016/j. arbres.2021.01.02

\section{CORRESPONDENCIA}

Cristhian Adolfo Vizcarra Vizcarra

cristhianvizcarral2@hotmail.com

ORCID: https://orcid.org/0000-0003-1068-7793

Fuente de financiamiento: por el autor.

Conflicto de interés: ninguno, según el autor.

Contribuciones de autoría: El autor concibió y diseñó el manuscrito, la recolección y análisis de los datos, la redacción y revisión crítica del contenido y la aprobación versión final que se publicará, asumiendo la responsabilidad de todos los aspectos del manuscrito.

Fecha de recepción: |8-03-202|.

Fecha de aceptación: 24-05-202I. 